\title{
Absolute Eigenvalues-Based Covariance Matrix Estimation for a Sparse Array
}

\author{
Kaushallya Adhikari
}

\begin{abstract}
The ensemble covariance matrix of a wide sense stationary signal spatially sampled by a full linear array is positive semi-definite and Toeplitz. However, the direct augmented covariance matrix of an augmentable sparse array is Toeplitz but not positive semi-definite, resulting in negative eigenvalues that pose inherent challenges in its applications, including model order estimation and source localization. The positive eigenvalues-based covariance matrix for augmentable sparse arrays is robust but the matrix is unobtainable when all noise eigenvalues of the direct augmented matrix are negative, which is a possible case. To address this problem, we propose a robust covariance matrix for augmentable sparse arrays that leverages both positive and negative noise eigenvalues. The proposed covariance matrix estimate can be used in conjunction with subspace based algorithms and adaptive beamformers to yield accurate signal direction estimates.
\end{abstract}

Index Terms-Covariance matrix, DOA estimation, positive semi-definite, sparse array, Toeplitz

\section{INTRODUCTION}

Estimation of directions of arrival (DOAs) of wide-sense stationary (WSS) signals using the data sampled by an array of sensors is an important task in many fields such as sonar, radar, seismology, radio astronomy, and neurophysiology. The data sampled by an $L$-element uniform linear array (ULA) that has an intersensor spacing of $\lambda / 2$, where $\lambda$ is the wavelength of the WSS signals impinging on the array, can be used to resolve up to $L-1$ DOAs [1], [2]. There exist augmentable sparse arrays for which the number of resolvable DOAs exceeds the number of sensors. An $L_{S}$-element augmentable sparse array that has an aperture of $\left(L_{F}-1\right) \lambda / 2$ can resolve $L_{A}-1$ DOAs where $L_{F} \geq L_{A} \geq L_{S}$. Sparse arrays where $L_{A}=L_{F}$ are called fully augmentable arrays and sparse arrays where $L_{S}<L_{A}<L_{F}$ are called partially augmentable arrays. Minimum redundant arrays [3] and nested arrays [4] are examples of fully augmentable sparse arrays. Semi-coprime arrays [5] are examples of partially augmentable sparse arrays. Coprime arrays [6] may be partially or fully augmentable based on the design parameters.

The accuracy and resolution of DOA estimates for any array also depends on the estimation algorithm. Conventional beamforming-based algorithms such as product processing and min processing have lower resolution than algorithms that are based on estimates of second order statistics (signal covariance matrix) such as multiple signal classification (MUSIC) [7]

This work was supported by the U.S. Office of Naval Research under Grant N00014-20-1-2820. The author is with the University of Rhode Island. e-mail: kadhikari@uri.edu or minimum variance distortionless response (MVDR) beamformer $[8]-[16]$ for augmentable sparse arrays. This work focuses on the algorithm that uses signal covariance estimates.

The performance of a DOA estimation algorithm such as MUSIC and MVDR depends on the accuracy of the covariance matrix estimate. The ensemble covariance matrix associated with an impinging WSS signal possesses two important properties: positive semi-definite and Toeplitz structure. Many covariance matrix estimates found in the literature do not possess one or both of these properties. For instance, the sample covariance matrix (SCM) of a full ULA (to be defined in Section III, which is widely used in DOA estimation, is positive semi-definite but is not guaranteed to be Toeplitz. The direct augmented matrix (DAM) of a sparse array (to be defined in Section III is Toeplitz but is not guaranteed to be semi-definite [17], [18]. For sparse arrays, Abramovich et al. have presented an algorithm for estimating a positive definite Toeplitz covariance matrix, called positive eigenvalues-based matrix (PEM) [17]. This algorithm generates reasonable covariance matrix estimates when the corresponding DAM has at least one nonnegative noise eigenvalue. However, when the DAM's noise eigenvalues are all negative, this algorithm is inapplicable. We present a new algorithm to estimate covariance matrix for sparse arrays that works even when all noise eigenvalues of the DAM are negative. Morever, when the proposed covariance matrix is used in conjunction with subspace based methods such as MUSIC [7] or adaptive algorithms such as MVDR beamformer [19], the DOA estimates are more accurate or commensurate with the PEM.

\section{Problem Formulation}

Consider an $L_{S}$-sensor linear array oriented along the positive $z$-axis. The sensor locations along the $z$-axis are $d_{1}=0$, $d_{2} \lambda / 2, d_{3} \lambda / 2, \ldots, d_{L_{S}} \lambda / 2=\left(L_{F}-1\right) \lambda / 2$, where $d_{2}, d_{3}, \ldots$, $d_{L_{S}}$ are assumed to be positive integers. Note that a ULA is a special case of this $L_{S}$-sensor array where $d_{2}=1, d_{3}=2$, $\ldots, d_{L_{S}}=L_{S}-1$. If the data sampled by the array consist of $Q$ WSS uncorrelated planewaves in white Gaussian noise, the received data vector at time $t$ is given by

$$
\mathbf{x}(t)=\sum_{i=1}^{Q} \mathbf{v}\left(\theta_{i}\right) s_{i}(t)+\mathbf{n}(t),
$$

where $s_{i}(t)$ is the complex amplitude of the $i^{t h}$ signal, $\mathbf{v}\left(\theta_{i}\right)$ is the array manifold vector corresponding to the $i^{t h}$ signal, $\theta_{i}$ is the $i^{t h}$ planewave angle measured from the positive $z$-axis, and $\mathbf{n}(t)$ is the $L_{S}$-element noise vector. We assume that the 
variables $s_{i}(t)$ and $\mathbf{n}(t)$ are zero-mean proper complex Gaussian random variable and random vector, respectively. The $k^{t h}$ element of the array manifold vector $\mathbf{v}\left(\theta_{i}\right)$ is $\exp \left(j \pi u_{i} d_{k}\right)$, where $u_{i}=\cos \left(\theta_{i}\right)$ is the direction cosine.

The SCM corresponding to the data vector in (1) is $\mathbf{x}(t) \mathbf{x}(t)^{H}$. Since the SCM is the outer product of a vector with itself, it is guaranteed to be positive semi-definite. If there are $T$ snapshots sampled at times $t=t_{1}, t_{2}, \ldots, t_{T}$, respectively, the SCM is given by the average of the data vectors' outer products, $\mathcal{S}=\sum_{k=1}^{T} \mathbf{x}\left(t_{k}\right) \mathbf{x}^{H}\left(t_{k}\right) / T$. The dimension of $\mathcal{S}$ is $L_{S} \times L_{S}$. To obtain a Toeplitz estimate, $\mathcal{T}$, we can apply redundancy averaging, which entails replacing every element of $\mathcal{S}$ by the average of the elements along the diagonal in which it resides. Since the dimension of $\mathcal{T}$ is $L_{S} \times L_{S}$, it can be used to estimate up to only $L_{S}-1$ DOAs. Sparse arrays such as MRAs, coprime arrays, and nested arrays are popular for their ability to resolve more DOAs than the number of sensors, which cannot be realized with $\mathcal{S}$ or $\mathcal{T}$. To utilize all degrees of freedom offered by a sparse array, consider the direct augmented matrix (DAM) which is obtained as described below:

label=,leftmargin $=*$

- Step 1: Find the possible covariance estimate at each possible lag as $r_{(0)}[k]=y[k] \star y^{*}[-k]$, where the symbol $\star$ denotes discrete convolution and $y[k]$ is the measurement made by the sensor at $z=k \lambda / 2$. If there is no sensor present at $z=k \lambda / 2, y[k]$ is considered 0 . The covariance function $r_{(0)}[k]$ is defined for lags in the range $-\left(L_{F}-1\right) \leq k \leq L_{F}-1$. However, some of the values of $r_{(0)}[k]$ are 0 in the range $-\left(L_{F}-1\right) \leq k \leq L_{F}-1$ if the sparse array is only partially augmentable.

- Step 2: Extract the hole-free covariance function estimate, $r_{(1)}[k]$ by truncating $r_{(0)}[k]$. This step is not needed in a fully augmentable sparse array. The function $r_{(1)}[k]$ is defined for lags in the range $-\left(L_{A}-1\right) \leq k \leq L_{A}-1$.

- Step 3: Remove the redundancy in $r_{(1)}[k]$ by discarding the values corresponding to negative lags. We will refer to the new covariance function as $r_{(2)}[k]$, which is defined for lags in the range $0 \leq k \leq L_{A}-1$.

- Step 4: Remove the bias in $r_{(2)}[k]$ to obtain $r_{(3)}[k]$ as $r_{(2)}[k] / c[k]$, where $c[k]$ is the hole-free coarray of the sparse array corresponding to positive lags.

- Step 5: The DAM is obtained by forming a Toeplitz matrix that has the elements $r_{(3)}[k]$ for $k=0,1, \ldots$, $L_{A}-1$ along its first row. This matrix is denoted by $\mathcal{T}_{D A M}$ in the sequel.

The DAM can be directly used with eigenanalysis based DOA estimation algorithms such as MUSIC [20], [21]. However, the DAM is not guaranteed to be positive semi-definite [17], [18].

\section{Positive Eigenvalues-Based Matrix}

An iterative algorithm to estimate a positive definite covariance matrix of dimension $L_{A} \times L_{A}$ for a sparse array is described in [17]. This algorithm uses the alternating convex projection method in [22] to compute an augmented covariance matrix that is both Toeplitz and positive definite. The algorithm keeps alternating between enforcing Toeplitz property and positive definiteness until the algorithm converges to a matrix that is both Toeplitz and positive definite. We summarize the essential components of their algorithm below: label=,leftmargin $=*$

- Step 1: Set $\mathcal{T}_{D A M}$ as the initial estimate of the covariance matrix: $\hat{\mathbf{T}}^{(0)}=\mathcal{T}_{D A M}$.

- Step 2: If $\hat{\lambda}_{1}, \hat{\lambda}_{2}, \ldots, \hat{\lambda}_{L_{A}}$ are the eigenvalues of $\hat{\mathbf{T}}^{(0)}$ in descending order, form the positive definite Hermitian matrix $\hat{\mathbf{H}}$ corresponding to $\hat{\mathbf{T}}^{(0)}$ as

$$
\hat{\mathbf{H}}=\bar{\lambda}_{n} \mathbf{I}_{L_{A}}+\sum_{j=1}^{Q}\left(\hat{\lambda}_{j}-\bar{\lambda}_{n}\right) \mathbf{v}_{j} \mathbf{v}_{j}^{H}
$$

where

$$
\bar{\lambda}_{n}=\frac{1}{L_{A}-Q} \sum_{j=Q+1}^{L_{A}} \hat{\lambda}_{j}
$$

is the average of the noise eigenvalues and $\mathbf{I}_{L_{A}}$ is the $L_{A} \times L_{A}$ identity matrix.

- Step 3: Given the positive definite matrix $\hat{\mathbf{H}}$, form the Toeplitz matrix $\hat{\mathbf{T}}^{(1)}$ by applying redundancy averaging.

- Step 4: Let $\hat{\lambda}_{1}, \hat{\lambda}_{2}, \ldots, \hat{\lambda}_{L_{A}}$ be the eigenvalues of $\hat{\mathbf{T}}^{(1)}$ in descending order. If $\frac{\hat{\lambda}_{Q+1}-\hat{\lambda}_{L_{A}}}{\hat{\lambda}_{L_{A}}} \geq \epsilon<<1$, where $\epsilon$ is a small positive number, then replace $\hat{\mathbf{T}}^{(0)}$ in Step $\mathbf{1}$ by $\hat{\mathbf{T}}^{(1)}$ and repeat Step 2, 3, and $\mathbf{4}$.

This algorithm converges when the largest noise eigenvalue is approximately equal to the smallest noise eigenvalue. [17] mentions that some of the noise eigenvalues of the DAM might be negative. To tackle this problem, [17] suggests discarding the negative noise eigenvalues and modifying (3) as

$$
\bar{\lambda}_{n}=\frac{1}{\sum_{j=Q+1}^{L_{A}} I[j]} \sum_{j=Q+1}^{L_{A}} \hat{\lambda}_{j} I[j],
$$

where $I[j]=1$ if $\hat{\lambda}_{j} \geq 0$ and $I[j]=1$ if $\hat{\lambda}_{j}<0$.

\section{A. Degenerative Case for PEM}

One important possibility that has not been addressed in [17] is that every noise eigenvalue of the DAM of a sparse matrix may be negative. The algorithm in [17] is not applicable when all noise eigenvalues are negative because it relies on having a positive subset of the noise eigenvalues to obtain $\bar{\lambda}_{n}$ in (4). To illustrate this point, we consider a fully augmentable sparse array depicted in Fig. 1. It is a coprime array formed by interleaving Subarray 1 that has 4 sensors and an intersensor spacing of $3 \lambda / 2$ and Subarray 2 that has 5 sensors and an intersensor spacing of $2 \lambda / 2$. The number of sensors in the array is 7 and the aperture is $9 \lambda / 2$. The corresponding DAM is of dimension $10 \times 10$. Consider a scenario with two planewave sources, each of SNR $25 \mathrm{~dB}$. The source direction cosines are selected as $u_{1}=0.2165 \frac{4}{10}$ and $u_{2}=-0.2165 \frac{4}{10}$ so that the source locations are half-power beamwidth apart for a full ULA with $9 \lambda / 2$ aperture [1, p. 999]. We used 25 snapshots to compute the DAM and find the corresponding eigenvalues. Note that the number of negative eigenvalues differ from trial to trial. The eigenvalues of the DAM were found to be 2603.9, 
$294.9,-10.2,-6.9,-6,-3.7,-3.1,-2.1,-1.7$, and -1.2 in one of the trials. The first two eigenvalues are signal plus noise eigenvalues and are positive. The remaining 8 eigenvalues are noise eigenvalues and all values are negative. A set of all negative noise eigenvalues poses an inherent challenge in covariance matrix estimation. In the next section, we propose a covariance matrix estimate to address this challenge.

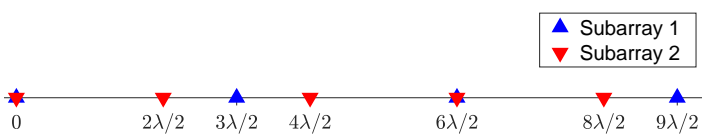

Fig. 1. A fully augmentable coprime array formed by interleaving Subarray 1 that has 4 sensors and an intersensor spacing of $3 \lambda / 2$ and Subarray 2 that has 5 sensors and an intersensor spacing of $2 \lambda / 2$.

\section{Absolute Eigenvalues-Based Matrix}

When the noise eigenvalues of the DAM are all negative, it renders the alternating convex projection method of covariance estimation inapplicable. We propose a method to estimate the augmented covariance matrix that leverages negative noise eigenvalues instead of discarding them. The proposed covariance matrix estimate is referred to as absolute eigenvaluesbased matrix (AEM). To motivate utilization of negative eigenvalues, consider the coprime array in Fig. 1 in the two planewave scenario described in Section III-A We approximated the probability density functions (PDFs) of two random variables: (1) minimum positive eigenvalue, $\min \left(\lambda^{(+)}\right)$, of the DAM; and (2) minimum of the absolute values of the negative eigenvalues, $\min \left(\left|\lambda^{(-)}\right|\right)$. These PDFs were obtained using $1,000,000$ realizations of the DAM, with each realization consisting of 10 snapshots. The PDFs are depicted in Fig. 2 for $0 \mathrm{~dB}$ and $10 \mathrm{~dB}$ SNR. The close resemblance between the PDFs of $\min \left(\lambda^{(+)}\right)$and $\min \left(\left|\lambda^{(-)}\right|\right)$suggests that negative noise eigenvalues can be used in improving the value of $\bar{\lambda}_{n}$. Therefore, given the eigenvalues of the DAM in descending order of their magnitudes $\left|\hat{\lambda}_{1}\right| \geq\left|\hat{\lambda}_{2}\right| \geq, \ldots, \geq\left|\hat{\lambda}_{L_{A}}\right|$, we propose calculating average noise eigenvalues as

$$
\bar{\lambda}_{n}=\frac{1}{L_{A}-Q} \sum_{j=Q+1}^{L_{A}}\left|\hat{\lambda}_{j}\right| .
$$

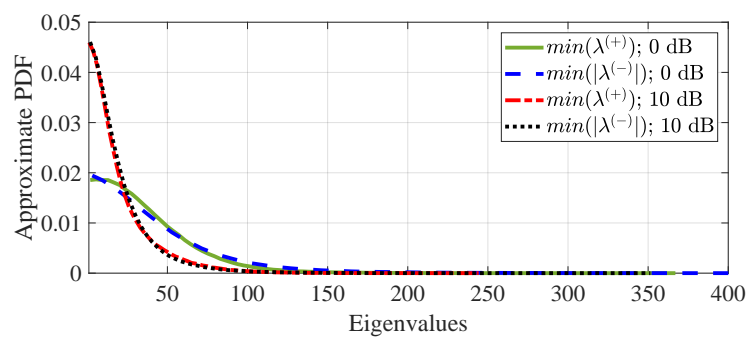

Fig. 2. Comparison of normalized histograms (approximated PDFs) of the minimum positive noise eigenvalue $\left(\min \left(\hat{\lambda}^{(+)}\right)\right)$and the minimum absolute value of negative noise eigenvalue $\left(\min \left(\left|\hat{\lambda}^{(-)}\right|\right)\right)$at two different $\mathrm{SNR}$ values.
The AEM is then formed as

$$
\boldsymbol{R}=\sum_{j=1}^{Q} \hat{\lambda}_{j} \mathbf{v}_{j} \mathbf{v}_{j}^{H}+\sum_{j=Q+1}^{L_{A}} \bar{\lambda}_{n} \mathbf{v}_{j} \mathbf{v}_{j}^{H},
$$

where $\mathbf{v}_{j}$ is the eigenvector corresponding to $\left|\lambda_{j}\right|$. Since each term in the two sums of (6) is a vector's outer product with itself, scaled by a non-negative scalar, $\mathcal{R}$ is guaranteed to be positive semi-definite. This approach does not fail when all noise eigenvalues are negative. Moreover, when only some of the noise eigenvalues are negative, using (5) provides an estimate of $\bar{\lambda}_{n}$ that has less variance since it is obtained by averaging over more realizations compared to (4). Note that $\mathcal{R}$ in (6) is not guaranteed to be Toeplitz. We can alternate between Toeplitz and positive semi-definite estimates following the approach used in the PEM computation. However, our simulation results in Section $\nabla$ show that $\mathcal{R}$ in $(6)$ is robust and does not warrant further improvement with an iterative method.

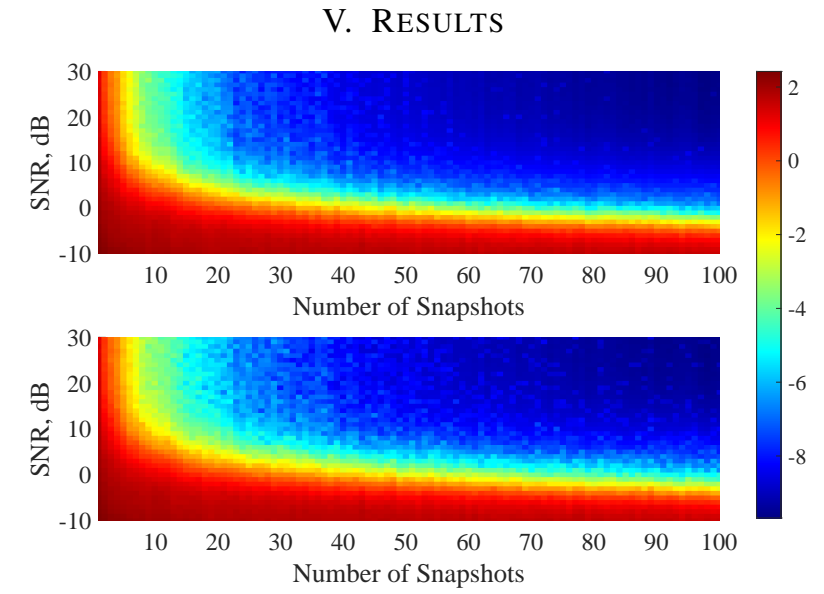

Fig. 3. Comparison of RMSEs for MUSIC using PEM (top panel) and AEM (bottom panel).

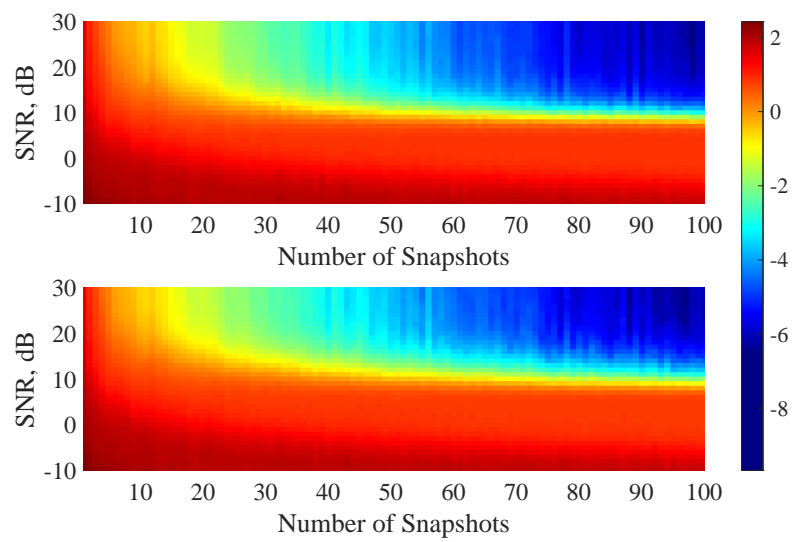

Fig. 4. Comparison of RMSEs for MVDR using PEM (top panel) and AEM (bottom panel)

This section compares the DOA estimation performance of MUSIC and MVDR using the covariance matrices PEM and AEM. We use the array in Fig. 1 in the two uncorrelated planewave scenario described in Section III-A. For fair comparison between the PEM and AEM based DOA estimation, 

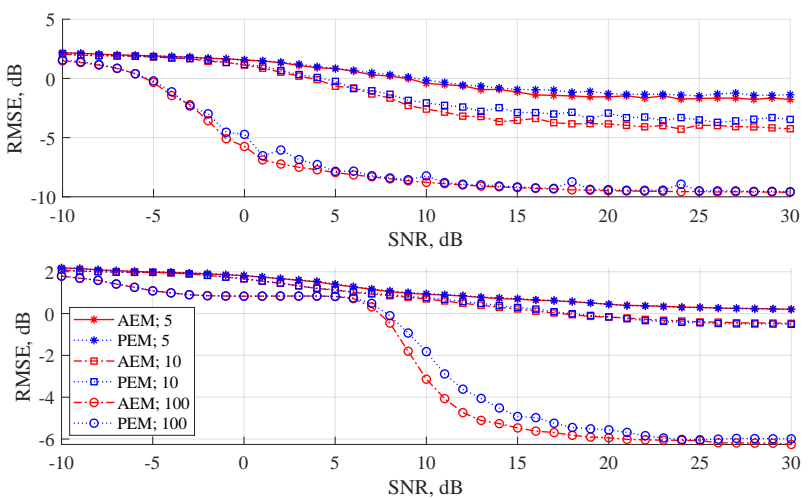

Fig. 5. Comparison of RMSEs in DOA for MUSIC (top panel) and MVDR (bottom panel) when AEM (red lines) and PEM (blue lines) are used as covariance matrix estimates.

we used the same 1000 datasets, discarding any dataset that consisted of all negative noise eigenvalues since the PEM cannot be evaluated for such datasets.

Fig. 3 compares the root mean squared error (RMSE) in $\mathrm{dB}$ scale for DOA estimation with MUSIC using PEM and AEM over a range of SNRs and numbers of snapshots. The top panel corresponds to the PEM-based MUSIC and the bottom panel corresponds to the AEM-based MUSIC. Comparing the two panels, we can infer that there is negligible difference between AEM and PEM based MUSIC, in cases when PEM is not degenerative. Similarly, Fig. 4 compares the RMSE with MVDR using PEM (top panel) and AEM (bottom panel). For MVDR also, there is no noticeable difference in the two panels. In Fig. 5, we plot several cuts of Fig. 3 and Fig. 4. We consider three different numbers of snapshots: 5 (less than the number of sensors), 10 (equal to the number of sensors), and 100 (greater than the number of sensors). The solid linestyle with asterisk marker indicates 5 snapshots, the dash-dot linestyle with square markers indicates 10 snapshots, the dotted line with circle marker indicates 100 snapshots; and the red and blue colors indicate AEM and PEM, respectively. From these RMSE plots, we can infer that in the cases when PEM is evaluable, the AEM-based estimation is either as accurate as or more accurate than the PEM-based estimation. This is true for both MUSIC and MVDR, which demonstrates that AEM is more robust than PEM.

\section{CONCLUSION}

We presented a robust positive semi-definite covariance matrix, AEM, for sparse arrays. The proposed approach does not degenerate when all eigenvalues of the DAM are negative. As evidenced by the simulations, the AEM is more robust than the competing covariance matrix estimate, PEM.

\section{REFERENCES}

[1] H. Van Trees, Optimum Array Processing (Detection, Estimation and Modulation Theory, Part IV). John Wiley and Sons, Inc., New York, 2002.

[2] D. Johnson and D. Dudgeon, Array Signal Processing: Concepts and Techniques. Simon \& Schuster, 1992.
[3] A. Moffet, "Minimum-redundancy linear arrays," IRE Trans. Antennas Propag., vol. 16, pp. 172-175, March 1968.

[4] P. Pal and P. Vaidyanathan, "Nested arrays: A novel approach to array processing with enhanced degrees of freedom," IEEE Transactions on Signal Processing, vol. 58, no. 8, pp. 4167 -4181, August 2010.

[5] K. Adhikari, "Beamforming with semi-coprime arrays," The Journal of the Acoustical Society of America, vol. 145, no. 5, pp. 2841-2850, 2019. [Online]. Available: https://doi.org/10.1121/1.5100281

[6] P. Vaidyanathan and P. Pal, "Sparse sensing with co-prime samplers and arrays," IEEE Transactions on Signal Processing, vol. 59, no. 2, pp. 573-586, February 2011.

[7] R. Schmidt, "Multiple emitter location and signal parameter estimation," IEEE Transactions on Antennas and Propagation, vol. 34, no. 3, pp. 276-280, March 1986.

[8] K. Adhikari, J. Buck, and K. Wage, "Extending coprime sensor arrays to achieve the peak side lobe height of a full uniform linear array," EURASIP Journal on Advances in Signal Processing, vol. 2014, no. 1, p. 148, Sep 2014. [Online]. Available: https: //doi.org/10.1186/1687-6180-2014-148

[9] G. Di Martino and A. Iodice, "Coprime synthetic aperture radar (copsar): A new acquisition mode for maritime surveillance," IEEE Transactions on Geoscience and Remote Sensing, vol. 53, no. 6, pp. 3110-3123, June 2015.

[10] K. Adhikari and B. Drozdenko, "Design and statistical analysis of tapered coprime and nested arrays for the min processor," IEEE Access, vol. 7, pp. 139601-139615, 2019.

[11] Y. Liu and J. R. Buck, "Gaussian source detection and spatial spectral estimation using a coprime sensor array with the min processor," IEEE Transactions on Signal Processing, vol. 66, no. 1, pp. 186-199, Jan 2018.

[12] K. Adhikari and J. Buck, "Gaussian signal detection with product arrays," IEEE Access, vol. 7, pp. 36 256-36266, 2020.

[13] K. Adhikari, J. Buck, and K. Wage, "Beamforming with extended coprime sensor arrays," 2013 IEEE International Conference on Acoustics, Speech and Signal Processing (ICASSP), pp. 4183-4186, May 2013.

[14] V. Chavali, K. Wage, and J. Buck, "Multiplicative and min processing of experimental passive sonar data from thinned arrays," journal $=$ The Journal of the Acoustical Society of America, vol. 144, no. 6, pp. 32623274, December 2018.

[15] K. Adhikari and J. Buck, "Gaussian signal detection by coprime sensor arrays," 2015 IEEE International Conference on Acoustics, Speech and Signal Processing (ICASSP), pp. 2379-2383, April 2015.

[16] I. M. Rooney and J. R. Buck, "Spatial power spectral density estimation using a welch coprime sensor array processor," The Journal of the Acoustical Society of America, vol. 145, no. 4, pp. 2350-2362, 2019. [Online]. Available: https://doi.org/10.1121/1.5097572

[17] Y. I. Abramovich, D. A. Gray, A. Y. Gorokhov, and N. K. Spencer, "Positive-definite toeplitz completion in doa estimation for nonuniform linear antenna arrays. i. fully augmentable arrays," IEEE Transactions on Signal Processing, vol. 46, no. 9, pp. 2458-2471, 1998.

[18] K. Adhikari and J. Buck, "Spatial spectral estimation with product processing of a pair of colinear arrays," IEEE Transactions on Signal Processing, vol. 65, no. 9, pp. 2389-2401, May 2017.

[19] J. Capon, "High-resolution frequency-wavenumber spectrum analysis," Proceedings of the IEEE, vol. 57, no. 8, pp. 1408-1418, Aug 1969.

[20] K. Adhikari and B. Drozdenko, "Symmetry-imposed rectangular coprime and nested arrays for direction of arrival estimation with multiple signal classification," IEEE Access, vol. 7, pp. 153 217-153 229, 2019.

[21] - "Comparison of music variants for sparse arrays," 2019 IEEE National Aerospace and Electronics Conference (NAECON), pp. 398405, 2019.

[22] K. M. Grigoriadis, A. E. Frazho, and R. E. Skelton, "Application of alternating convex projection methods for computation of positive toeplitz matrices," IEEE Transactions on Signal Processing, vol. 42, no. 7, pp. 1873-1875, 1994. 